\title{
HIDRÓLISE DO ÓLEO DE CRAMBE UTILIZANDO AGITADOR ORBITAL
}

\author{
D. MOLINARI ${ }^{1}$, E. RAIZER ${ }^{1}$, J. A AWADALLAK ${ }^{1}$,R. L. S. CANEVESI ${ }^{1}$, T. O. \\ REINEHR $^{1}$, L. C. PAVAN ${ }^{1}$, E. A. SILVA ${ }^{1}$ \\ ${ }^{1}$ Universidade Estadual do Oeste do Paraná, Departamento de Engenharia Química
}

\begin{abstract}
RESUMO - Os combustíveis no Brasil têm sido produzidos em grandes escalas, gerando empregos e promovendo crescimento econômico e segurança energética. Biodiesel é um combustível alternativo ao diesel de petróleo, feito a partir de fontes renováveis tais como, óleos vegetais e gorduras de origem animal que reagem com um alcool na presença de catalisadores. A hidroesterificação é uma das rotas de produção de biodiesel e que tem sido muito investigada, consistindo em uma etapa de hidrolise seguida de esterificação. Estas reações quando realizadas com catalisadores heterogêneos, necessitam de altas temperaturas e pressões para ocorrer, o que pode ser contornado com o uso de enzimas como catalisador, as quais utilizam condições mais brandas. $\mathrm{O}$ crambe é uma oleaginosa que possui condições agronômicas favoráveis e uma considerável fonte de óleo não comestível. Este trabalho teve como objetivo investigar a reação de hidrólise enzimática do óleo de crambe, visando a produção de ácidos graxos livres, empregando a lipase Lecitase Ultra (Fosfolipase A1), em reator batelada com agitação orbital. Um planejamento de delineamento central do composto rotacional (DCCR) foi empregado para estudar os efeitos das variáveis, temperatura, fração água/óleo (A/O) e fração de enzima/substrato $(\mathrm{E} / \mathrm{S}$, onde, $\mathrm{S}=$ massa de total de água e óleo), no rendimento da reação que foi acompanhada a partir da análise de acidez. O rendimento foi de 65,36\%, durante 12 horas de reação à $50^{\circ} \mathrm{C}$.
\end{abstract}

\section{INTRODUÇÃO}

O esgotamento das fontes de energia não renováveis, como petróleo e o carvão, tem impulsionado novas pesquisas por combustíveis derivados de fontes renováveis de energia. Como exemplo tem-se o biodiesel, um combustível não fóssil, renovável, atóxico, o qual pode substituir total ou parcialmente o diesel de petróleo em motores de ciclo diesel (GAMA et al., 2010). A produção e o uso do biodiesel no Brasil propiciam o desenvolvimento de uma fonte energética sustentável sob os aspectos ambiental, econômico e social e também trazem a perspectiva da redução das importações de óleo diesel. (ANP, 2014). O processo mais comum de obtenção de biodiesel é a transesterificação, que se refere a uma reação química catalisada, envolvendo óleo vegetal e um álcool para se obter ésteres alquílicos de ácidos graxos como produto (isto é, o biodiesel) e o glicerol como subprotudo (ZHANG et al., 2003). Geralmente na presença de um catalisador homogêneo ou heterogêneo (GAMA et al., 2010).

Recentemente vem sendo estudado também o processo de hidroesterificação, que envolve uma etapa de hidrólise seguida de uma etapa de esterificação. A hidrólise consiste em 
uma reação química entre a gordura, ou o óleo, com a água, gerando glicerina e ácidos graxos livres, aumentando propositadamente a acidez da matéria-prima. Após a hidrólise, os ácidos graxos gerados são então esterificados com um álcool, que "neutraliza" a acidez presente. $\mathrm{O}$ biodiesel produzido por hidroestrificação é gerado com elevada pureza, sem necessidade de etapas de lavagem, que geram efluentes e elevados consumo de compostos químicos. $\mathrm{Na}$ reação, também é possível obter como subproduto a água, que retorna para o processo de hidrólise (ENCARNAÇÃO, 2008).

Entre os processos, a hidrólise enzimática apresenta vantagens econômicas e técnicas, pois pode ser realizada em condições amenas $\left(20\right.$ a $40^{\circ} \mathrm{C}$ e pressão atmosférica), levando a um menor gasto energético em comparação aos processos físico-químicos (RITTNER, 1996). A enzima líquida comercial, Lecitase Ultra (Fosfolipase A1) é postulada para as posições 1,3 nos triglicerídeos, devido à sua especificidade (WANG et al., 2010).

Uma das formas de reduzir a dependência de óleo comestível para fazer biodiesel é usar óleos vegetais não comestíveis (LUQUE \& MELERO, 2012). O crambe (Crambe abyssinica) possui potencial considerável como fonte de óleo para a produção de biodiesel, demonstrando bom rendimento e produção de óleo por hectare por ano (MACHADO et al., 2011). Um aspecto importante é que o óleo de crambe, devido a sua alta concentração de ácido erúcico, substitui derivados de petróleo na fabricação de muitos produtos e como ele é um produto renovável, tem a seu favor um grande apelo ambiental que valoriza o produto (PITOL et al., 2012).

Este trabalho tem como objetivo avaliar a reação de hidrólise enzimática do óleo de crambe em reator batelada com agitação orbital, assim como a influencia de algumas variáveis do processo, utilizando a lipase Lecitase Ultra (Fosfolipase A1).

\section{MATERIAIS E MÉTODOS}

\subsection{Materiais}

O óleo de crambe utilizado como substrato da hidrólise nesse trabalho foi fornecido pela Fundação MS $\otimes_{\circledast}$. A enzima líquida comercial Lecitase Ultra, utilizada como catalisador enzimático, foi gentilmente cedida por LNF® Latino Americana. Álcool Etilico (Chemco®), Éter etílico (Anidrol®) e NaOH (Synth®), foram utilizados para determinação da acidez. Como indicador da solução de titulação, foi utilizada uma solução de fenolftaleína (Synth $\left.{ }^{\circledR}\right)$.

O aparelho de agitação orbital (TECNAL TE-421) foi utilizado na reação de hidrólise para a mistura dos reagentes, contidos em erlenmayers de $50 \mathrm{~mL}$. A separação de fases foi realizada em uma centrífuga (Parsec ${ }^{\circ}$ CT-0603).

\subsection{Métodos}

Reações de hidrólise: As reações de hidrólise enzimática do óleo de crambe conduzidas em um aparelho de agitação orbital do tipo shaker a $180 \mathrm{rpm}$, foram realizadas com o intuito de analisar a influência das variáveis, temperatura, fração mássica de água /óleo e fração mássica de enzima/substrato exercem sobre a acidez. As quantidades de reagentes utilizados foram de $10 \mathrm{~g}$ de óleo fixados e massas variáveis de água deionizada e de enzima, determinada de acordo com o planejamento experimental, assim como a temperatura. Ao término de cada tempo de reação, as amostras foram retiradas do aparelho e aquecidas até uma temperatura de cerca de $100{ }^{\circ} \mathrm{C}$ em um aparelho de micro ondas com o intuito de 
desativar a enzima, interrompendo a reação e diminuindo a viscosidade do meio. A amostra era então centrifugada (Centrifuga PARSEC CT-0603) a 3 g durante 5 minutos, com o intuito de separar a fase aquosa contendo a enzima e o óleo para posterior análise de acidez.

Quantificação da Acidez: A quantidade de ácidos graxos livres presentes no óleo de crambe foi determinada por titulação com uma solução de $\mathrm{NaOH} 0,05 \mathrm{M}$. Aproximadamente $1 \mathrm{~g}$ de amostra foi diluída em $25 \mathrm{ml}$ de solução isovolumétrica de álcool etílico e éter etílico com uma gota de fenolftaleína. A solução foi então titulada sob vigorosa agitação até que houvesse mudança de coloração para rosa, indicando a neutralização da acidez. A Equação (01) demonstra o cálculo da acidez:

$$
\operatorname{Acidez}(m \%)=100 . \frac{\text { Vol. } M_{\mathrm{NaOH}} \cdot P M_{A G L}}{p a}
$$

A massa molecular do AGL foi calculada como a média ponderada pela fração molar das massas moleculares dos ácidos graxos constituintes do óleo de crambe.

Planejamento experimental: Um planejamento experimental composto central rotacional 23 com triplicata no ponto central foi utilizado para avaliar o efeito das variáveis independentes: temperatura $\left({ }^{\circ} \mathrm{C}\right)$, fração água/óleo $(\mathrm{m} \%)$ e fração enzima/substrato $(\mathrm{m} \%)$ sobre a variável dependente: quantidade de ácidos graxos livres (AGL) em relação à massa total do produto final (\%).

Foram utilizados 17 tratamentos, sendo 8 fatoriais (combinações entre os níveis -1 e +1 ); 6 axiais (uma variável no nível $\pm 1,68$ e duas no nível 0 ); 3 centrais (todas variáveis no nível 0). Na Tabela 1 encontram-se os níveis da variáveis do planejamento experimental.

Tabela 1- Níveis de variáveis no planejamento experimental

\begin{tabular}{cccccc}
\hline Variáveis codificadas & $-1,68$ & -1 & 0 & +1 & $+1,68$ \\
\hline Temperatura $\left({ }^{\circ} \mathrm{C}\right)$ & 56,7 & 30 & 40 & 50 & 23,2 \\
$\begin{array}{c}\text { Fração água/óleo }(\mathrm{m} \%) \\
\begin{array}{c}\text { Fração enzima/substrato } \\
(\mathrm{m} \%)\end{array}\end{array}$ & 3,2 & 10 & 20 & 30 & 36,8 \\
\hline
\end{tabular}

\section{RESULTADOS E DISCUSSÃO}

A acidez das reações de hidrólise enzimática do óleo de crambe foi analisada apor meio de um processo de titulação. A Tabela 2 apresenta os resultados do planejamento para a hidrolise enzimática do óleo de crambe utilizando reator batelada com agitação orbital a uma rotação fixa em $180 \mathrm{rpm}$. 
Tabela 2- DCCR e acidez experimental para agitador orbital

\begin{tabular}{ccccc}
\hline Ensaio & $\begin{array}{c}\text { Temperatura } \\
\left(\mathbf{C}^{\mathbf{C}}\right)\end{array}$ & $\begin{array}{c}\mathbf{A} / \mathbf{O} \\
(\mathbf{m} \%)\end{array}$ & $\begin{array}{c}\mathbf{E} / \mathbf{S} \\
(\mathbf{m} \%)\end{array}$ & $\begin{array}{c}\text { Acidez } \\
(\mathbf{\%})\end{array}$ \\
\hline 1 & & & & \\
\hline 2 & 30 & 10,0 & 1,50 & 39,18 \\
3 & 30 & 10,0 & 4,50 & 45,66 \\
4 & 30 & 30,0 & 1,50 & 40,46 \\
5 & 30 & 30,0 & 4,50 & 50,30 \\
6 & 40 & 20,0 & 3,00 & 50,47 \\
7 & 40 & 20,0 & 3,00 & 50,73 \\
8 & 40 & 20,0 & 3,00 & 51,21 \\
9 & 50 & 10,0 & 1,50 & 50,03 \\
10 & 50 & 10,0 & 4,50 & 56,49 \\
$\mathbf{1 1}$ & 50 & 30,0 & 1,50 & 56,47 \\
12 & $\mathbf{5 0}$ & $\mathbf{3 0 , 0}$ & $\mathbf{4 , 5 0}$ & $\mathbf{6 5 , 3 6}$ \\
13 & 23,2 & 20,0 & 3,00 & 42,19 \\
14 & 56,7 & 20,0 & 3,00 & 55,36 \\
15 & 40 & 3,2 & 3,00 & 38,93 \\
16 & 40 & 36,8 & 3,00 & 56,81 \\
17 & 40 & 20,0 & 0,49 & 38,43 \\
& 40 & 20,0 & 5,50 & 59,16 \\
\hline
\end{tabular}

Para um período de 12 horas de reação o maior rendimento encontrado, que está apresentado em negrito na tabela acima, foi de $65 \%$ de ácidos graxos, na condição de temperatura de $50^{\circ} \mathrm{C}$ e quantidades de reagentes de $30 \%$ de fração água/óleo e $4,50 \%$ de fração enzima/substrato, com massa de óleo fixada em $10 \mathrm{~g}$.

Figura 1 - Diagrama de pareto para a produção de AGL, sob um intervalo de $95 \%$ de confiança.

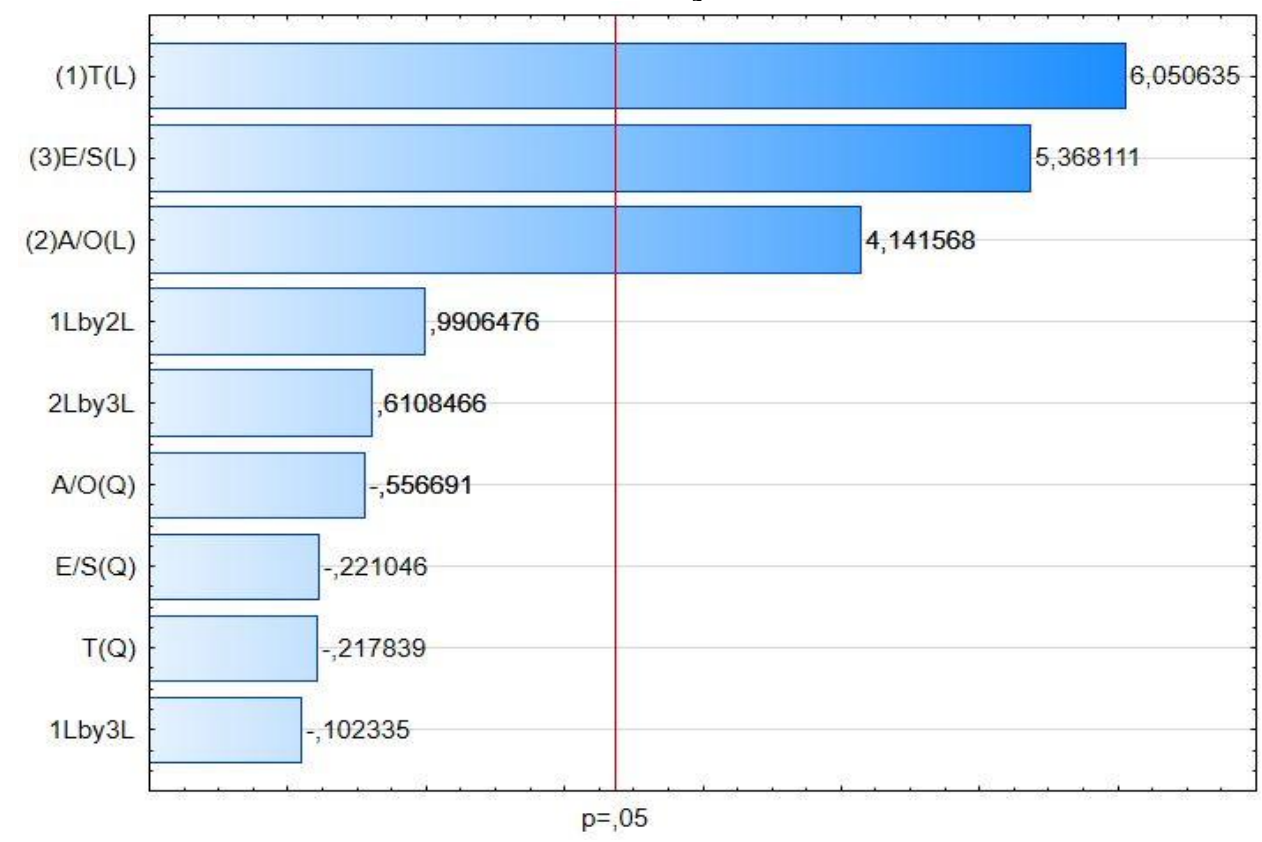


É possível observar no diagrama de pareto que a nível de significância de 95\%, os efeitos temperatura, fração enzima/substrato e fração água/óleo se mostraram relevantes (pvalor $<0,05)$.

A verificação da validade do modelo foi realizada pela análise do teste F. Quando Fcalc $>$ Ftab o modelo é válido e se ajusta bem aos dados experimentais. A Tabela 4 mostra a análise da variância para o delineamento bem como o valor de Ftab. Para um intervalo de confiança de $95 \%$, tem-se o valor tabelado de F: Ftab $(9 ; 7 ; 0,05)=3,68$.

Tabela 4- Análise de variância

\begin{tabular}{cccccc}
\hline $\begin{array}{c}\text { Fonte de } \\
\text { variação }\end{array}$ & SQ & GL & QM & $\mathbf{F}_{\text {calc }}$ & $\mathbf{F}_{\text {tab }}$ \\
\hline Regressão & 946,494 & 9,00 & 105,166 & 9,36 & 3,68 \\
Resíduos & 78,614 & 7,00 & 11,2306 & & \\
Total & 1025,108 & 16,00 & & & \\
\hline
\end{tabular}

Como Fcalc é maior que o Ftab a nível de 5\%, o modelo é válido e se ajusta bem aos dados experimentais. O coeficiente de regressão encontrado foi de 0,92331 , o que significa que a conversão em ácidos graxos como função do teor de água, enzima e temperatura, pode ser bem explicada pelo modelo apresentado pela Equação (05).

\section{CONCLUSÕES}

Neste trabalho foi estudada a hidrólise enzimática do óleo de crambe em reator batelada com agitação orbital e foi avaliado os efeitos das variáveis temperatura, fração água/óleo e enzima/substrato, no rendimento da reação.

Foi constatado com o uso do agitador orbital que o melhor rendimento $(65,36 \%)$ foi obtido em condições maiores de temperatura $\left(50^{\circ} \mathrm{C}\right)$ e quantidades maiores de reagentes $(30 \%$ de fração água/oleo e $4,50 \%$ de fração enzima/substrato) para um período de 12 horas. Constatando a importância de se estudar a hidrolise do óleo de crambe bruto a partir do uso de agitadores que trazem altas conversões, viabilizando o seu uso para a produção de biodiesel pela rota de hidroesterificação.

\section{NOMENCLATURA}

Acidez $=$ Fração mássica de AGL na amostra

$A G L=$ Ácido(s) Graxo(s) Livre(s)

Calc $=$ Calculado

$T a b=$ Tabelado

$M_{\mathrm{NaOH}}=$ Molaridade da solução de $\mathrm{NaOH}$ (mol 1-1)

$p a=$ Peso da amostra de óleo $(\mathrm{g})$

$P M_{A G L}=$ Peso molecular médio dos AGL presentes no óleo (g mol-1)

$V o l=$ Volume da solução de $\mathrm{NaOH}$ utilizado na titulação (l) 


\section{REFERÊNCIAS}

ANP - Agência Nacional do Petróleo, Gás Natural e Biocombustíveis. Biodiesel: Introdução, 2014. Disponível em: <www.anp.gov.br>. Acesso em: 26 de Março de 2015.

ENCARNAÇÃO, A. P. G. Geração de biodiesel pelos processos de transesterificação e hidroesterificação, uma avaliação econômica. Diss. Mestrado em Tec. de Proc. Quím. e Bioq. Rio de Janeiro: UFRJ/EQ, 2007.

GAMA, P. E.; GIL, R. A. S. S.; LACHTER, E. R. Produção de biodiesel através de transesterificação in situ de sementes de girassol Via catálise homogênea e heterogênea. Quím. Nova. Rio de Janeiro: PubliSBQ, 2010. v. 33, n. 9, p. 1859-1862.

LUQUE, R.; MELERO, J. A. Advances in biodiesel production: Processes and Technologies. Espain: Woodhead Publishing, 2012. p. 1- 8.

MACHADO, M. F.; BRASIL, A. N.; OLIVEIRA, L. S.; NUNES, D. L. Estudo do Crambe (Crambe Abyssinica) como fonte de óleo para produção de biodiesel. ENERBIO. Itaúna-MG: Núcleo de Pesq. e Desenv.em Café, Depart. de Eng. Quím., UFMG, 2011.

RITTNER, H. Óleo de palma: Processamento e utilização. São Paulo, 1ed. P. 311-320, 1996.

PITOL, C.; ROSCOE, R.; ERBES, E. J.; ROMEIRO, T. S.; SANTOS, J. F. Cultura do Crambe: Resultados e Experimentação. Tec. e Prod.: Milho Safrinha e Cult.de Inverno 2012. Maracaju-MS: Fundação MS, 2012.

WANG, W.; ZHAO, M.; SONG, K.; WANG, L.; TANG, S.; RILEY, W. W. Partial hydrolysis of soybean oil by phospholipase (Lecitase Ultra). Food Chem. China: Elsevier, 2010. v. 121, n. 4, p. 1066-1072.

ZHANG, Y.; DUBÉ, M. A.; MCLEAN, D. D. Biodiesel production from waste cooking oil: 1. Process design and technological assessment. Bioresource Technology. OttawaCanadá: Elsevier, 2003. v. 89, p. 1-16. 